\title{
A Case of Giant Right Atrium Diverticulum with Atrial Septal Defect
}

\author{
Yangming Fan, Hui Jiang, Yu Wei, Bing Zhang, Jianjun Ge \\ Department of Cardiovascular Surgery, The First Affiliated Hospital of USTC, Division of Life Sciences and Medicine, \\ University of Science and Technology of China, Hefei, Anhui, China
}

\section{ABSTRACT}

Right atrium diverticulum is a rare congenital malformation. We present a previously unreported case of giant right atrium diverticulum $(153 \times 109 \mathrm{~mm})$ in a 17 -year-old female patient. Echocardiographic examination also showed an atrial septal defect $(11 \mathrm{~mm})$. Considering the risk of right atrium rupture, we performed femoral arteriovenous cannulation first, followed by median thoracotomy. The defect was repaired with an autologous pericardial patch. The patient recovered well after the operation, and this case is referential for surgical treatment of giant right atrium diverticulum.

\section{INTRODUCTION}

Right atrial diverticulum (RAD) is a very rare cardiac anomaly, with etiology reported to be congenital [Gulati 2011; Binder 2000]. RAD is also termed right atrial aneurysm, diverticulum, or idiopathic dilatation [Narain 2012; Bezuska 2018]. Complications including arrhythmia, thrombosis, and rupture may occur. In these cases, the diverticulum may be found incidentally as an enlargement of the cardiac silhouette on chest radiographs, whereas severe cardiac insufficiency is quite rare. In the present case, the patient presented to the doctor with obvious mobility restrictions.

\section{CASE REPORT}

This study was approved by the Biomedical Ethics Committee of Anhui Medical University under protocol number ah56743 and is consistent with the Declaration of Helsinki.

In 2018, a 17-year-old female patient presented at our hospital with a heart murmur since childhood, which was found when she walked for the first time, and severe activity restriction for 3 months. The echocardiographic (ECG)

Received fanuary 30, 2021; received in revised form March 12, 2021; accepted March 12, 2021.

Correspondence: Bing Zhang, The First Affiliated Hospital of USTC, No. 17 Lujiang Road, Hefei, Anbui 230001, China; 8617756059814 (e-mail: bzhang@mail.ustc.edu.cn); and Fianjun Ge, The First Affiliated Hospital of USTC, No. 17 Lujiang Road, Hefei, Anbui 230001, China; 8613605693993 (e-mail: zkdgjj@ustc.edu.cn). examination in the outpatient department showed an atrial septal defect $(11 \mathrm{~mm})$, and a giant cystic structure $(153 \times 109$ $\mathrm{mm}$ ) was visible on the outside of the right heart. Part of the section was connected to the right atrium, blood flow in the dark cystic area was static, and the right heart was obviously compressed (Figure 1). The inner diameter of the left atrium was $25 \mathrm{~mm}$, and the end-diastolic inner diameter of the left ventricle was $33 \mathrm{~mm}$.

After the patient was admitted to the hospital, cardiac computed tomography angiography (CTA) examination revealed that the right atrium was abnormally enlarged (the larger cross-sectional area was approximately $139 \times 115 \mathrm{~mm})$, the right atrium wall was abnormally thin, and the entire left atrium and right ventricle were significantly compressed and shifted to the left and rear, prompting the diagnosis of right diverticulum (Figure 2). ECG showed sinus rhythm, abnormal $\mathrm{Q}$ waves in the anterior wall, low-voltage QRS waves in chest leads, and ST-T changes (Figure 3A). Considering that the right atrium diverticulum was extremely large and had obvious clinical manifestations, with severe compression of the left ventricular system and right ventricle, surgical treatment was planned.

After anesthesia was induced, we first performed retrograde cardiopulmonary bypass (CPB) with femoral artery and venous cannulation (CPB duration of 60 minutes, cooling from $37^{\circ}$ to $32.8^{\circ}$ in 1.5 minutes and maintained for 16 minutes, no cross-clamp, and hypothermic circulatory

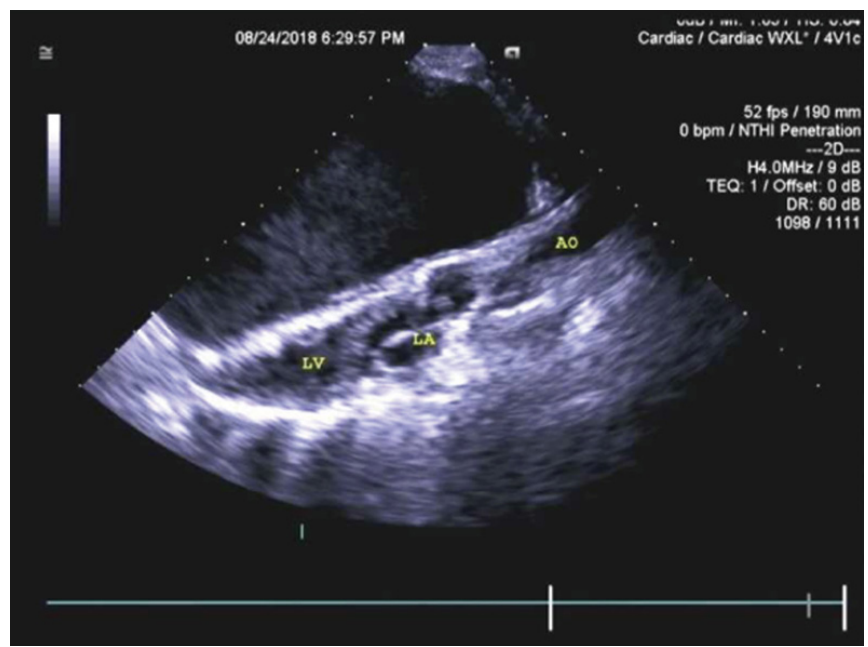

Figure 1. Two-dimensional echocardiogram showing large diverticulum (upper left corner) 
arrest) to empty the blood in the right atrium diverticulum. The traditional median approach was then performed to cannulate the superior vena cava. The right atrium diverticulum was open. The wall of the diverticulum was thin, rising from the lateral wall of the right atrium, and no thrombosis was observed. There was no obvious expansion of the proper atrium, and a defect of approximately $11 \mathrm{~mm}$ was observed in the middle segment of the atrial septum. The defect was repaired by continuous suture with an autologous pericardial patch.

Next, the diverticulum wall was cut along the boundary between the diverticulum and the normal atrial wall, and the right atrium and part of the diverticulum residual margin were continuously sutured with 5-0 Prolene thread. The opening in the right atrium was closed with a continuous 5-0 polypropylene suture.

The patient recovered well after the operation and was discharged 6 days later. Follow-up ECG was normal (Figure $3 \mathrm{~B})$, and measurements were $32 \mathrm{~mm}$ for the left atrium and 39
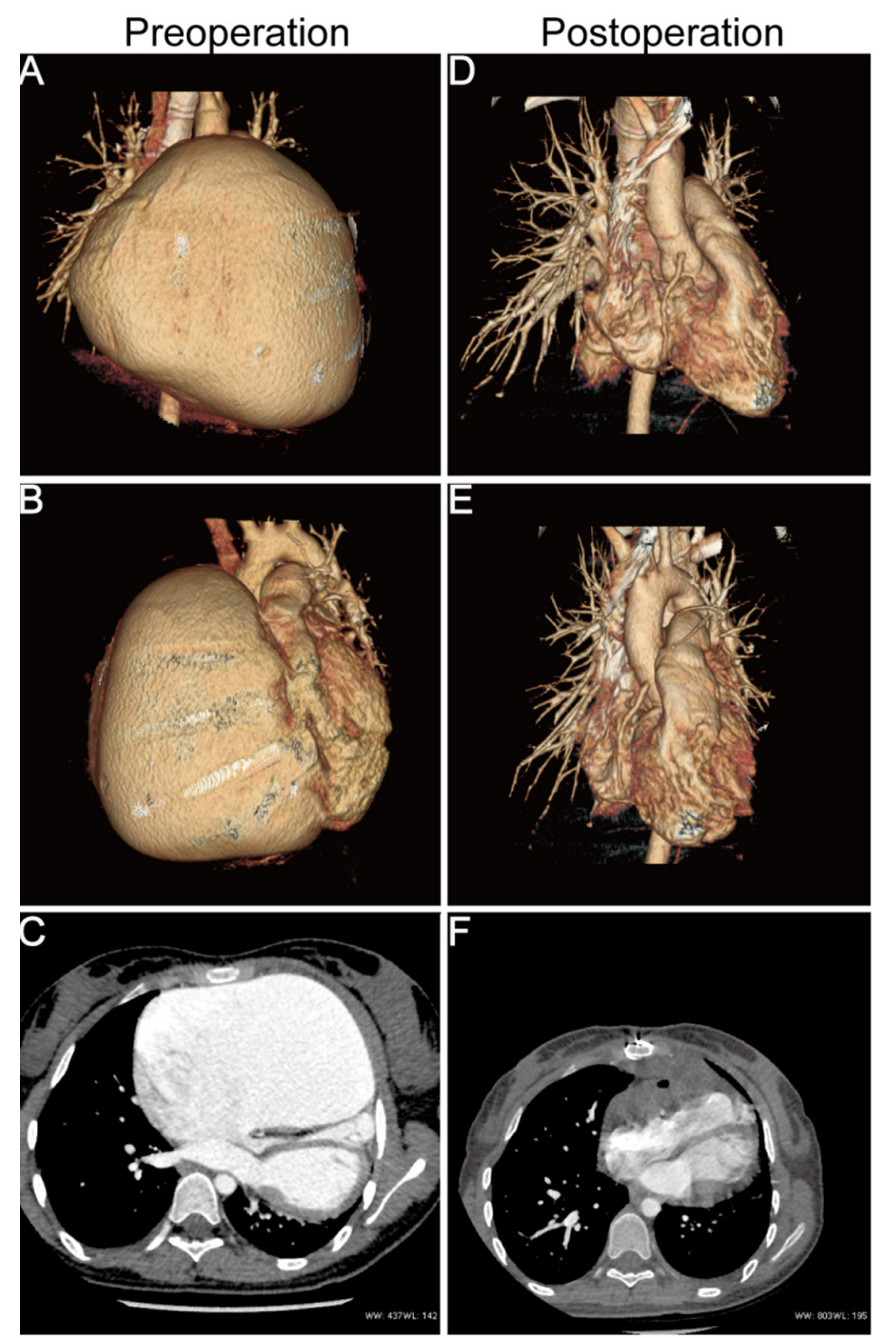

Figure 2. Computed tomography angiogram showing the large diverticulum before $(A, B$, and $C)$ and after $(D, E, F)$ surgery. $\mathrm{mm}$ for left ventricular end-diastolic diameter (significantly improved over preoperative size), and cardiac function was good. Follow-up CTA is shown in Figure 1. Postoperative histopathology showed no myocardial element in the wall of the diverticulum; it was being replaced by fibroconnective tissue with an endocardial lining (Figure 3C). On 1-year postoperative follow-up, cardiac physiology and function had

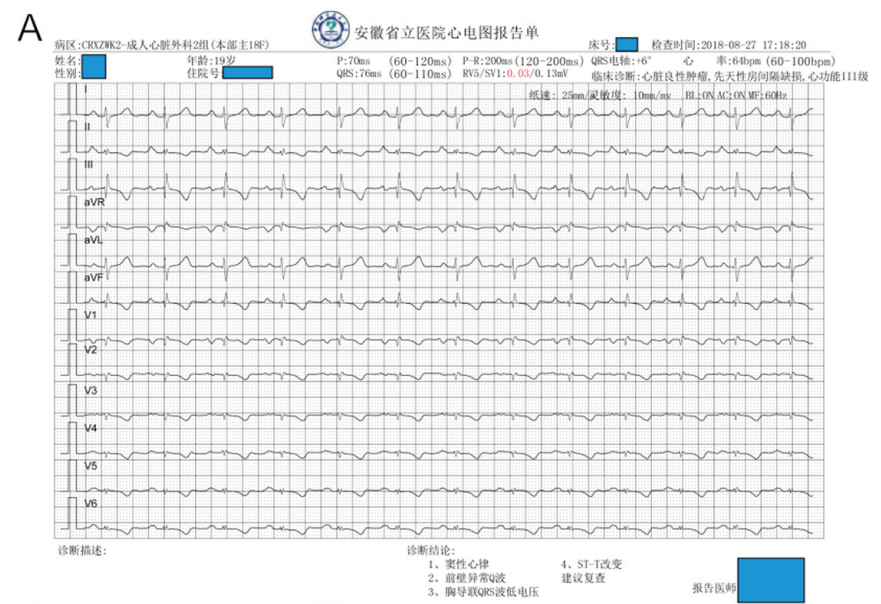

B

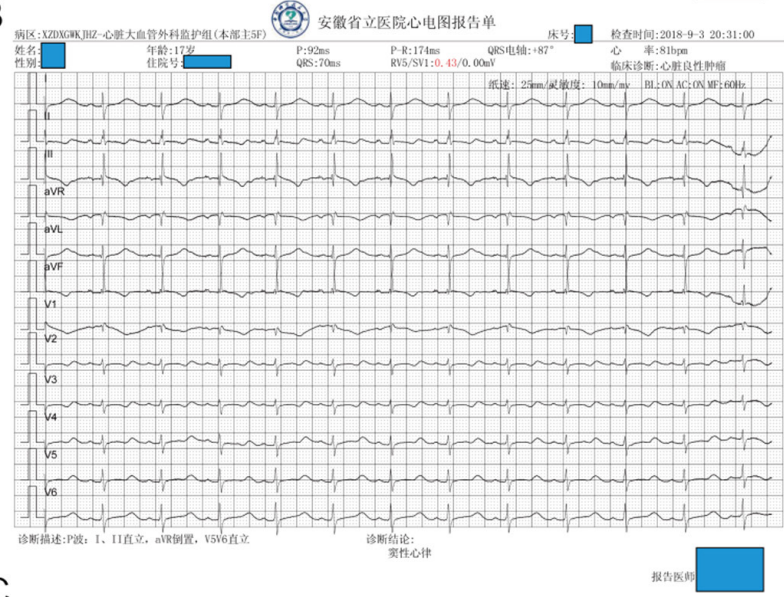

C

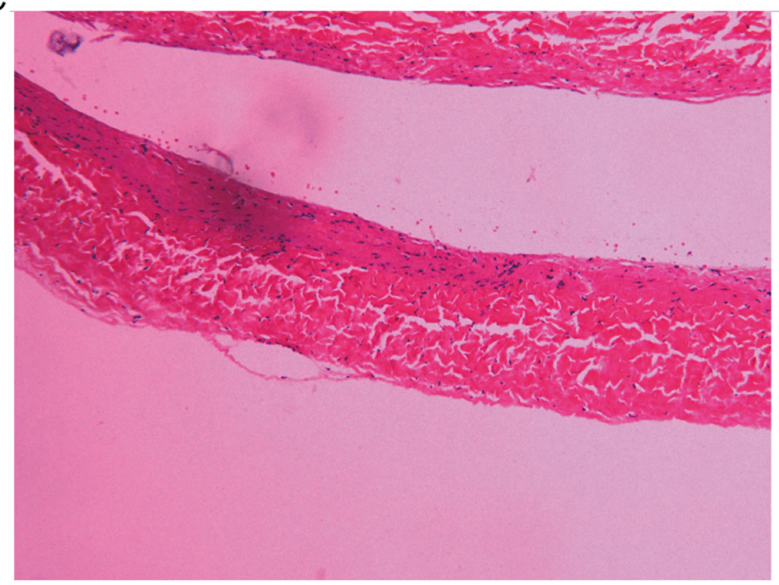

Figure 3. ECG result before (A) and after (B) surgery. C, Histopathology of the wall of the diverticulum showed that there was no myocardial element. 
returned to normal, with ECG measurements of $36 \mathrm{~mm}$ for the left atrium and $48 \mathrm{~mm}$ for the left ventricle.

\section{DISCUSSION}

Here, we report a patient with an atrial septal defect (11 $\mathrm{mm})$ and giant cystic structure $(153 \times 109 \mathrm{~mm})$ on the outside of the right heart. Considering the risk of right atrium rupture, we performed femoral arteriovenous cannulation first, followed by cutting off the RAD and repairing the atrial septal defect.

A single RAD is a congenital anomaly rarely reported worldwide, with age varying from fetus to adult [Borgohain 2013]. The etiology has not been clarified to date [Bezuska 2018; Borgohain 2013; Morrow 1968]. Lipomatous degeneration and reduction of muscular elements can be found in the wall of the diverticulum. Thus, Tenckhoff [1969] proposed that single RAD may be an Uhl's anomaly.

RAD patients usually come to the hospital because of gross cardiomegaly detected by routine chest radiographs. It is very rare to have severe mobility restriction as in the present case, which was mainly because the diverticulum was abnormally large. Previously reported RAD diameters rarely exceed 100 $\mathrm{mm}$. Such a huge cystic structure acutely compressed the right ventricle and the entire left ventricular system, resulting in severe cardiac insufficiency. Preoperative ECG confirmed that the left atrium and left ventricle were significantly smaller than normal because of compression. This patient also had an atrial septal defect of $11 \mathrm{~mm}$. A continued shunt from left to right may aggravate the dilatation of the diverticulum and the right atrium wall. There was no obvious atrial or supraventricular arrhythmia in the patient's preoperative course and ECG, but myocardial ischemia was still suggested to be present. The postoperative ECG was almost normal, implying that the abnormal electrical activity was related to RAD.

For asymptomatic patients with RAD, whether surgical intervention is needed is still controversial. But for symptomatic patients, surgical resection is unanimously recommended. In this case, the diverticulum was huge, and the wall was thin. The front wall of the diverticulum was close to the entire sternum. If a conventional median sternal split were performed and then cardiopulmonary bypass established, there would be risk of right atrium rupture. Thus, we decided to perform femoral arteriovenous cannulation first, followed by median thoracotomy. The results confirm that this strategy is indeed safe and reliable.
Unfortunately, we could not determine an accurate shape of the diverticulum with this strategy, but preoperative CTA reconstruction pictures show its remarkable nature. We believe that for a relatively large atrial diverticulum, surgical intervention should be considered with or without symptoms, because the risk of surgery is far lower than the risk of sudden death or other adverse events [Bezuska 2018]. Some atrial diverticulum may be repaired without extracorporeal circulation, but the surgery is safer and more reliable under extracorporeal circulation, especially in this case.

\section{ACKNOWLEDGMENTS}

This work was supported by the National Natural Science Foundation of China (grant number NSFC 81470530 to J. $\mathrm{Ge}$ ) and the Major Science and Technology Projects of Anhui (18030801132 to J. Ge).

\section{REFERENCES}

Bezuska L, Bu'Lock FA, Anderson RH, Speggiorin S, Corno AF. Giant right atrial aneurysm: Antenatal diagnosis and surgical treatment. World J Pediatr Congen Heart Surg 2018;9:459-462.

Binder TM, Rosenhek R, Frank H, Gwechenberger M, Baumgartner H. Congenital malformations of the right atrium and the coronary sinus: An analysis based on 103 cases reported in the literature and two additional cases. Chest 2000;117:1740-1748.

Borgohain S, Malik L, Prasad S, Gupta A, Grover V, Gupta VK. Case of single right atrial diverticulum and review of etiology and management. Asian Cardiovasc Thoracic Ann 2013;21:592-595.

Gulati A, Gheta R, Chan CF, Ismail NA, Sheppard MN, Kilner PJ, Magee AG. Longitudinal follow-up of a right atrial appendage aneurysm by cardiac magnetic resonance imaging. Circulation 2011;123:2289-2291.

Morrow AG, Behrendt DM. Congenital aneurysm (diverticulum) of the right atrium. Clinical manifestations and results of operative treatment. Circulation 1968;38:124-128.

Narain VS, Puri A, Sethi R, Aga P, Behera S, Ahmed N, Saran RK. Giant right atrial aneurysm presenting as right heart failure. Indian Heart J 2012;64:200-202.

Tenckhoff L, Stamm SJ, Beckwith JB. Sudden death in idiopathic (congenital) right atrial enlargement. Postmortem findings and review of cases. Circulation 1969;40:227-235. 